\title{
Direct-imaging of light-driven colloidal Janus particles in weightlessness
}

\author{
Cite as: Rev. Sci. Instrum. 91, 013902 (2020); doi: 10.1063/1.5124895 \\ Submitted: 18 August 2019 - Accepted: 13 December 2019 • \\ Published Online: 6 January 2020
}

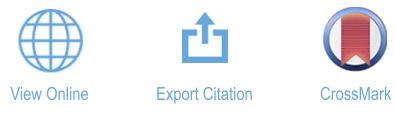

\author{
R. Keßler, ${ }^{1, a)}$ D. Bräuer, ${ }^{1}$ C. Dreißigacker, ${ }^{1}$ J. Drescher, ${ }^{1}$ C. Lozano, ${ }^{2}$ C. Bechinger, ${ }^{2}$ (D) P. Born, \\ and Th. Voigtmann ${ }^{1,3}$ (D)
}

\begin{abstract}
AFFILIATIONS
${ }^{1}$ Institut für Materialphysik im Weltraum, Deutsches Zentrum für Luft- und Raumfahrt (DLR), 51170 Köln, Germany

${ }^{2}$ University of Konstanz, 78457 Konstanz, Germany

${ }^{3}$ Department of Physics, Heinrich-Heine-Universität Düsseldorf, Universitätsstraße 1, 40225 Düsseldorf, Germany
\end{abstract}

Note: This paper is part of the Special Collection: Materials and Life Science Experiments for the Sounding Rocket MAPHEUS. a) raphael.kessler@dlr.de

\begin{abstract}
We describe a highly integrated automated experiment module that allows us to investigate the active Brownian motion of light-driven colloidal Janus-particle suspensions. The module RAMSES (RAndom Motion of SElf-propelled particles in Space) is designed for the sounding rocket platform MAPHEUS (MAterialPHysikalische Experimente Unter Schwerelosigkeit). It allows us to perform experiments under weightlessness conditions in order to avoid sedimentation of the Janus particles and thus to study the spatially three-dimensional dynamics in the suspension. The module implements a newly developed strong homogeneous light source to excite self-propulsion in the Janus particles. The light source is realized through an array of high-power light-emitting diodes and replaces the conventional laser source, thus reducing heat dissipation and spatial extension of the experiment setup. The rocket module contains ten independent sample cells in order to ease the systematic study of the effect of control parameters such as light intensity or particle concentration and size in a single sounding-rocket flight. For each sample cell, transmitted light intensities are stored for postflight analysis in terms of differential dynamical microscopy.
\end{abstract}

Published under license by AIP Publishing. https://doi.org/10.1063/1.5124895

\section{INTRODUCTION}

Suspensions of colloidal Janus particles are intriguing model systems to study out-of-equilibrium "active" soft matter, i.e., soft matter whose constituents possess mechanisms to convert energy into directed motion on the microscale. ${ }^{1-3}$ Janus particle suspensions consist of dispersed micrometer-sized particles that are anisotropic regarding their chemical, optical, or other properties; a prominent example is colloidal spheres whose one hemisphere is coated with a second material. ${ }^{4,5}$ The anisotropy can be used to induce an asymmetric drift through interaction with the solvent, by consuming some dissolved fuel or energy from an external supply. Hence, it drives a "self-propulsion" mechanism acting on each particle on top of the thermal Brownian motion. These self-propelled particles are a good experimental realization of active Brownian particles (ABPs), a theoretical model system that allows us to address generic physical effects of self-propulsion on the dynamics of colloidal particles. ${ }^{6}$ This is of specific interest in biophysics and biological applications, where ABPs help us to understand physical principles behind the emergent collective behavior of active matter, ${ }^{7-12}$ be it related to bacterial colonies or the motion of tissue cells in wound healing, the schooling of fish, the swarming of insects, or the flocking of birds. In particular, the clustering behavior of colloidal Janus particles due to their active motion has attracted a lot of attention under the heading of "motility-induced phase separation" (MIPS). . $^{211,13}$

To date, experimental studies on the stationary dynamics of model ABP suspensions have mostly been restricted to quasi-twodimensional situations. The suspension media have to be chosen to meet the requirements of the respective propulsion mechanism, leaving only little flexibility to density-match the particles. The particles thus sediment to the bottom of the sample cell, on time scales comparable to the MIPS processes. It is desirable to extend experiments to three-dimensional situations for a twofold reason: first, the dimensional dependence of phenomena such as MIPS should be clarified ${ }^{12}$ and second, specific hydrodynamically mediated 
interactions between the particles and the surface might introduce unwarranted side effects and thus obscure the analysis. ${ }^{14,15}$ Gravity can also cause polar order in otherwise spherically interacting active particles.

A way to perform experiments using well-established activeparticle suspension systems, but getting rid of gravity-driven effects, is to carry them out under microgravity conditions. In the following, we describe a setup called RAMSES (RAndom Motion of SElf-propelled particles in Space) that we have designed to enable such experiments on three-dimensional model ABP suspensions in microgravity. The setup is designed for the sounding rocket MAPHEUS (MAterialPHysikalische Experimente Unter Schwerelosigkeit; materials physics experiments under weightlessness) as a highly integrated and autonomous measurement system. It enables in particular the direct comparison of the dynamics of model $\mathrm{ABP}$ suspensions in quasi-two-dimensional situations on ground and in three dimensions in microgravity. The setup was flown on the MAPHEUS-07 campaign (launched in February 2018 from Esrange, Sweden) and allowed experiments during approximately 6 min of weightlessness conditions. A modified version of the module, containing improved sample-cell handling and better optical alignment, was flown as part of the MAPHEUS-08/ATEK campaign (launched in June 2019).

RAMSES is based on a previous on-ground laboratory setup, but it introduces several enhancements and features that were customized for the sounding-rocket environment. In particular, a new light source was developed based on arrays of high-power lightemitting diodes (LEDs) with a specifically adapted optical system to provide homogeneous illumination of the sample cells. The LED array sources replace the laser source used in the laboratory setting and significantly reduce power consumption and heat dissipation during the experiment, which is crucial for the realization of a highly integrated setup. This allows us to contain ten sample cells, each provided with independent illumination and detection units, providing thermostatization to within $\pm 0.5^{\circ} \mathrm{C}$, in the confined space of a single sounding-rocket module. In addition, a new sample cell layout was developed for 3D samples and also adapted to compensate for the strong acceleration and centrifugal forces that act on the suspensions during the startup phase of the rocket flight. The sample-cell environments are contained in two lateaccess chambers and can be operated remotely through an Ethernet connection.

In the present paper, we describe the details of the RAMSES flight hardware together with an overview of the postflight data processing procedure. We give an overview of the requirements from the experimental system and the sounding-rocket environment in Secs. II A and II B, respectively. The hardware of RAMSES is described in Sec. III, and exemplary data analysis is shown in Sec. IV. We conclude in Sec. V.

\section{SPECIFICATIONS}

\section{A. Experimental system}

Our experimental model system for ABPs consists of colloidal silica spheres, coated on one hemisphere with a carbon layer (Janus particles) and suspended in a binary mixture of water and lutidine. ${ }^{17,18}$ A typical particle size is $2.8 \mu \mathrm{m}$ with a coating of an estimated thickness of $20 \mathrm{~nm}$. In the suspension, the particles follow mainly repulsive interactions.

The binary solvent is chosen such that at temperatures below a critical point, $T<T_{c} \approx 34{ }^{\circ} \mathrm{C}$ for the given composition, the fluid mixture remains homogeneous, while above $T_{c}$, demixing sets in. The sample is maintained at a target temperature just below $T_{c}$; in our setup, we use $T=28^{\circ} \mathrm{C} \pm 0.5^{\circ} \mathrm{C}$.

The particles' caps strongly absorb electromagnetic radiation in the visible range, to which both the particles' cores and the solvent are nearly transparent. Thus, illuminating the sample with a suitable strong light source leads to a local heating of the caps, which, in turn, causes a local demixing of the solvent in the vicinity of the caps. As a result, an anisotropic driving mechanism acts on the particles to propel them along the axis of asymmetry defined by the capped hemisphere. The details of the driving mechanism are not important for the present purpose, although they are an interesting topic of study in their own right. It suffices here that empirically wellcharacterized self-propulsion forces can be set up in the system by absorption of light ${ }^{19}$ and tuned by the temperature distance to the demixing critical point, $\Delta T=T_{c}-T$, and the light intensity.

The model system has been used in numerous on-ground experiments ${ }^{13}$ studying the quasi-2D motion. Specifically, in a suitable density and light-intensity range, the suspensions are found to undergo MIPS. ${ }^{2}$

Due to the density mismatch between the particles $\left(\rho_{m} \approx 2.65\right.$ $\left.\mathrm{g} / \mathrm{cm}^{3}\right)$ and the solvent $\left(\rho_{m} \approx 0.98 \mathrm{~g} / \mathrm{cm}^{3}\right)$, the particles quickly sediment under the action of gravity. Since the specific choice of solvent is dictated by the mechanism to implement self-propulsion of the particles, density matching of the particles is difficult to achieve. Previous studies of the system were thus confined to the quasi-2D motion close to the bottom of the sample cell.

\section{B. Sounding rocket environment}

MAPHEUS is a sounding rocket program set up by the Institute of Materials Physics in Space of the German Aerospace Center (DLR-MP, Cologne), jointly with the Mobile Rocket Base (MORABA) team of the DLR Institute of Space Operations and Astronaut Training, Oberpfaffenhofen. In its current design, each rocket carries several independent experiments each housed in 17in. modules designed to provide a self-sustained experiment environment, including own power supply and automated control and monitoring. The rocket carries a service module that provides each experiment with some relevant signals, such as lift-off.

The MAPHEUS-07 rocket was launched on February 17, 2018, from the Skylark tower at Esrange, Sweden. The rocket was about $11.3 \mathrm{~m}$ high and carried about $365 \mathrm{~kg}$ of payload. Using two engine stages, an apogee of about $247 \mathrm{~km}$ above ground was reached, allowing for approximately $357 \mathrm{~s}$ under weightlessness conditions. After re-entering the Earth's atmosphere, it landed using a parachute system and the payload modules were recovered by a helicopter. MAPHEUS-08 was launched on June 13, 2019, carrying $379 \mathrm{~kg}$ payload and reached an apogee of about $239 \mathrm{~km}$ providing $360 \mathrm{~s}$ of weightlessness conditions.

During the startup phase, the sounding rocket undergoes very strong vertical acceleration. The modules thus have to be designed to withstand up to $40 \mathrm{~g}$ equivalent static load. In addition, the flight path of the rocket is initially stabilized by a spin with a 
frequency of approximately $4 \mathrm{~Hz}$ around the vertical axis. The resulting centrifugal forces can cause accumulation of the colloidal particles on the outward side of the sample cells and thus have to be compensated.

During preparation and the launch procedure, the rocket is placed in the launcher for an a priori unknown extended period of time. To accommodate samples that are not long-term stable (e.g., due to solvent evaporation or sticking of the colloidal particles to the cell walls), late access is provided during the countdown phase, about $2 \mathrm{~h}$ before lift-off.

\section{RAMSES MODULE SETUP}

Figure 1 shows the design of the RAMSES module. It is composed of an outer module (yellow) that is part of the rocket structure and two sample chambers that are inserted into the outer module and that each contain five in principle identical sample environments. Each sample environment consists of a sample cell in the middle, an illumination device at the bottom, and an imaging lens and a camera at the top, connected to a Raspberry Pi computer. The complete setup fits in a 17 -in. module of $400 \mathrm{~mm}\left(153 / 4^{\prime \prime}\right)$ height and has a weight of $43 \mathrm{~kg}$ overall.

The outer module consists of a support structure including rails to guide the two late-access inserts, a power supply including 9

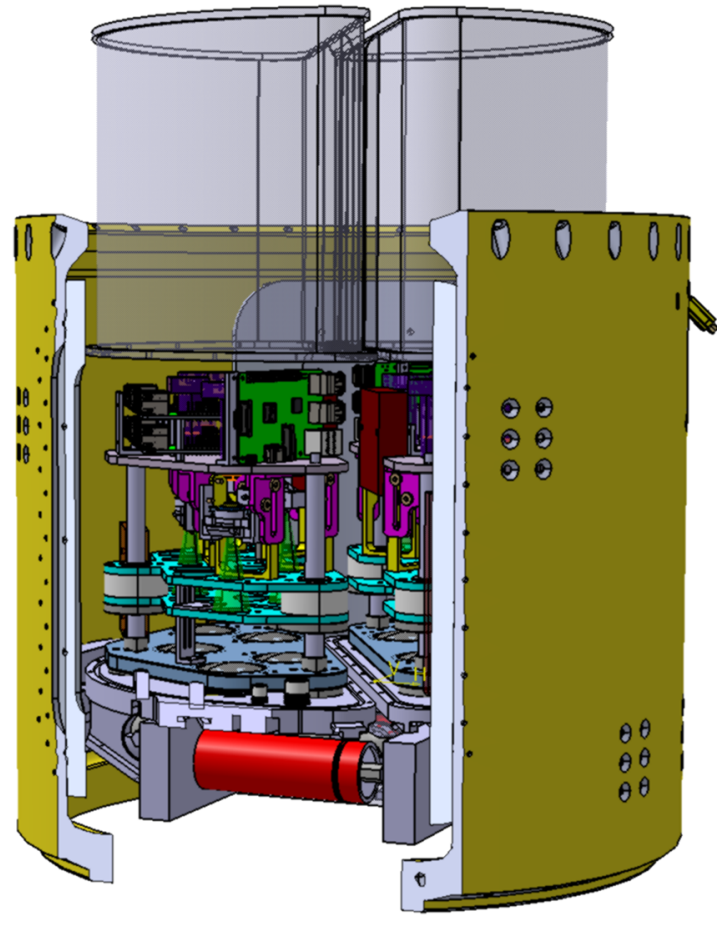

FIG. 1. CAD drawing of the RAMSES sounding rocket module for the MAPHEUS07 sounding rocket. The outer part of the module (yellow) contains structural elements and the power supply (red) and allows for two module inserts to be inserted through hatches to provide late-access facility. Two identical module inserts containing five sample environments each are used. Each insert is an atmospheric pressure chamber (hoods shown vertically lifted for clarity).
LiFePo4 batteries (Headway 38120HP, $3.2 \mathrm{~V}$ nominal voltage, $8 \mathrm{Ah}$ capacity), and appropriate charging electronics. The batteries provide a power supply of $28.8 \mathrm{~V}$ with a peak current of $I_{\text {peak }}=160 \mathrm{~A}$. At a nominal load current of $80 \mathrm{~A}$, the onboard power supply provides an electrical power of $2304 \mathrm{~W}$ and a capacity of $0.23 \mathrm{kWh}$. The main electrical load is generated by the LED array $(70$ LEDs in total operating at $3.3 \mathrm{~V}$ and using $0.7 \mathrm{~A}$, drawing a total power of $161.7 \mathrm{~W}$ ), the Raspberry Pi computers ( $50 \mathrm{~W}$ maximum power for all ten), and the Peltier elements used in the temperature control of the samples (QuickCool QC-32-0.6-1.2 operating at 3.9 V and $1.2 \mathrm{~A}$, drawing $4.7 \mathrm{~W}$ each at a cooling power of $1.6 \mathrm{~W}$, thus $47 \mathrm{~W}$ in total over all ten cells if heating/cooling is active). The spindle motor used to lift the illumination units into place after entering the microgravity phase consumes $39.2 \mathrm{~W}$. In total, the peak power consumption of the module, when all electrical consumers are running, amounts to just over $300 \mathrm{~W}$, which amounts to about $0.03 \mathrm{kWh}$ per experimental run (assuming a 6-min microgravity phase). During standby, the module has a power consumption of about $36 \mathrm{~W}$. Umbilicals are connected to an external power supply to power the experiment and keep the batteries charged prior to lift-off. A further umbilical provides an Ethernet connection to the sample chambers in order to allow remote interaction and monitoring until lift-off.

The two sample chambers are pressurized to provide ambientpressure conditions during the entire flight phase. This facilitates the use of standard electronic equipment inside the chamber and prevents enhanced solvent evaporation. The sample chambers are in principle identical to each other. Each of them is inserted at an angle of $30^{\circ}$ to the pitch axis through one of the two dedicated hatches (cf. Fig. 2), manually locked, and connected to the Ethernet umbilical. Connecting the chambers to the power supply and signal plug automatically starts up the experiment control and prepares the experiments to start automatically upon lift-off. The countdown procedures for the MAPHEUS- 07 and MAPHEUS- 08 campaigns permitted to insert the late-access chambers $2 \mathrm{~h}$ before lift-off. This allowed filling the sample cells approximately $4 \mathrm{~h}$ before the experiment start; this time period is sufficiently short to maintain sample stability.

Each sample chamber consists of five sample cell environments, schematically depicted in Fig. 3. For each, illumination is provided by an array of high-power LEDs described below, whose light is

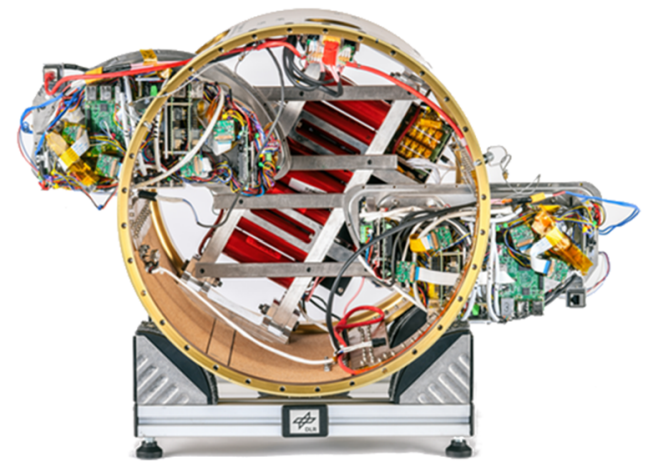

FIG. 2. RAMSES module viewed from the top, with the two late-access inserts half-way inserted, omitting their atmospheric-pressure chamber covers for clarity. 


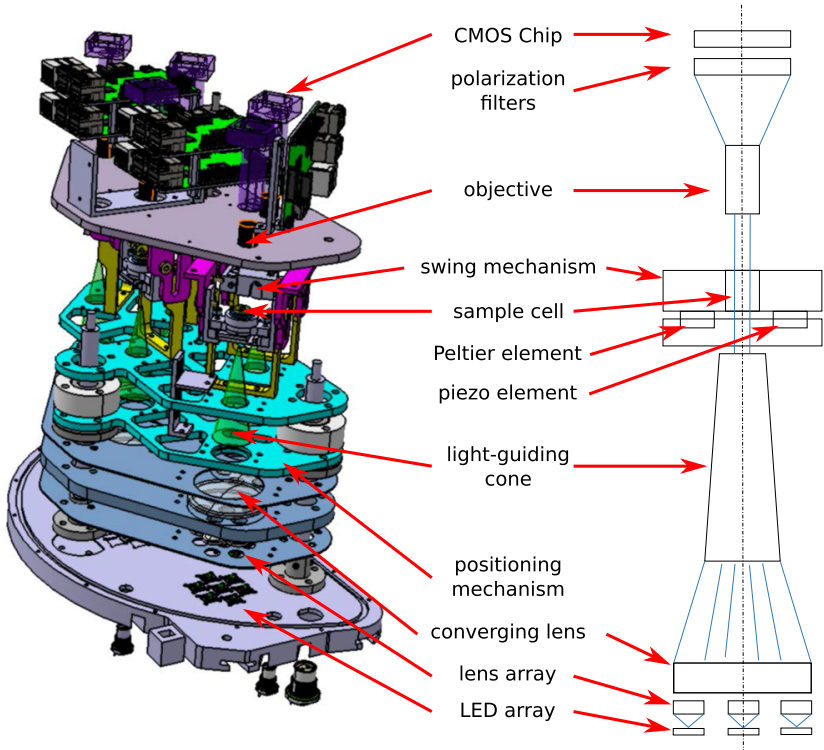

FIG. 3. Schematic drawing of a single RAMSES probe environment. Five identical probe environments are combined on a common baseplate to form one of the two module inserts. Illumination for imaging and driving the particles is provided by an LED array (bottom) whose light is homogenized and directed to the sample cell by a light cone and moved in place by a positioning mechanism at the beginning of the microgravity phase of the flight. Transmitted light is, after suitable filtering, detected by using a CMOS camera.

coupled into the sample cell through a light-guiding system that directly attaches to the bottom of the sample cell in flight configuration. As described below, the sample cells are provided with a swing mechanism that serves to provide well-defined acceleration forces during the lift-off phase and to counteract centrifugal forces caused by the rocket spin. These swings are locked into place in the microgravity-flight configuration using the light cone and a mechanical guiding mechanism.

For each sample cell, the light detection unit contains an objective, polarizers for intensity adjustment, and a camera to record the transmitted light intensity. Each camera is controlled by using a dedicated Raspberry Pi mini-computer running Linux, which also controls the experiment and stores the recorded images from the camera. The Pi computers for four of the sample environments are connected to an Ethernet switch to allow remote control through the umbilical connection. This feature was used during the countdown phase mainly for manual temperature control and a visual verification of sample homogeneity through a live view of the camera picture. The actual experimental protocol does not require manual intervention; in fact, one out of the five Raspberry computers is not connected to the Ethernet switch due to space constraints inside the pressure chamber.

The general-purpose input/output (GPIO) connectors of the Pi computers are used to connect the temperature control for each sample cell and signals from the service module so that the computer routines can detect the lift-off signal given by that service module. One of the Pi computers serves as a master, also controlling a shaker for the sample cells and a spindle system to lock the sample cells in a predefined position after the launch phase (see below).

\section{A. Illumination for self-propulsion}

We have developed a strong LED illumination system that is capable of delivering the high homogeneous light intensity that is needed to incite self-propelled motion of the particles in the suspension. This avoids the use of a laser source and allows us to minimize the space required by the individual sample setup. For imaging purposes, the LED light source with limited coherence is also beneficial for the analysis in terms of dynamical differential microscopy (DDM). ${ }^{21-2}$

To achieve the desired intensities, seven high-power LEDs (Osram LT CP7P) are bundled for each sample cell. The LEDs are placed on an aluminum bottom plate that acts as a heat sink. The light from the LED arrays is collected by using specifically designed collimator lenses (a lens array of one lens per LED) and an additional large converging lens covering the whole array (focal length $f=50 \mathrm{~mm}$ ). The lenses were cast and polished from epoxy resin. The collimated light is homogenized by a cylindrical cone with a capped top (turned from polymethylmethacrylate and polished) that guides the light and attaches to the bottom of the sample cell in flight configuration.

The principle of this light homogenization optics is that within the cone, the light rays from the converging lens are internally reflected multiple times with increasingly steep angles such that the exit angles at the top of the cone are effectively randomized and spatially homogeneous light is provided. For this, the cone is placed a small distance above the converging lens such that its bottom diameter $(D=25 \mathrm{~mm})$ matches the size of the light patch coming from the LED array. The top diameter of the cone, $d=3 \mathrm{~mm}$, is slightly larger than that of the sample cell, in order to cut off unavoidable intensity peaks at the fringes. The length $L$ of the cone then sets its opening angle, and in principle, a larger length causes more reflections and thus better expected homogeneity, but also larger loss due to a larger area near the top of the cone where the conditions for total internal reflections are no longer met. In practice, the height $(L=70 \mathrm{~mm})$ is fixed by the available vertical space in the setup.

The intensity spectrum of the LED array (shown in Fig. 4) displays a clear maximum around a wave length of $\lambda \approx 524 \mathrm{~nm}$.

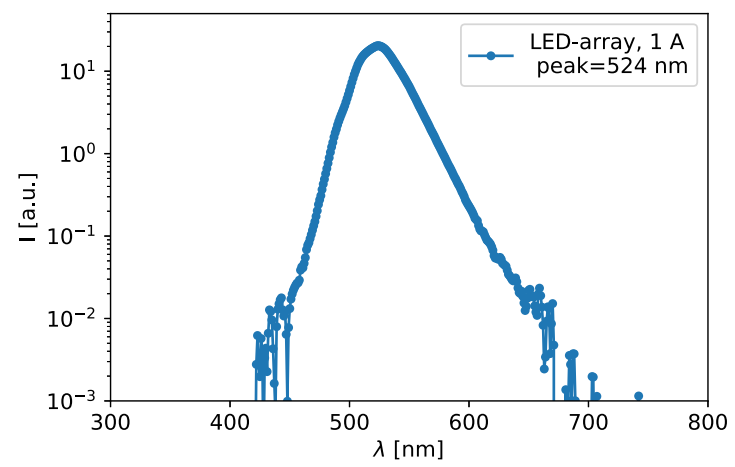

FIG. 4. Intensity spectrum of the LED array developed for RAMSES, at a driving current of $1 \mathrm{~A}$. 
This corresponds well to the laser source previously used in the onground experiment $(\lambda \approx 532 \mathrm{~nm})$ and fits well in the frequency band where the carbon caps of the Janus particles strongly absorb electromagnetic radiation, but the solvent and the particles themselves do not. Hence, self-propulsion through local heating of the caps is triggered. The spectrum of the LED array is broader than that of the conventional laser source, but for the purpose of both driving self-propulsion and illumination of the sample, this broadening of the spectrum is irrelevant. We further checked that the light intensity is stable over the duration of a typical flight experiment and that intensity fluctuations are small (less than $2 \%$ standard deviation) if operated at typical currents of $1 \mathrm{~A}$. These intensity characteristics are reached in the first approximately $30 \mathrm{~s}$ after switching on the array, which is sufficiently quick for in-flight operation.

We have checked the spatial homogeneity of the sample-cell illumination, which is important to avoid systematic spatial variations in the self-propulsion force. For this, an exemplary light source was taken and profiled using a Westboro Photonics P501U photometer (focal length $f=50 \mathrm{~cm}$, exposure time $64 \mathrm{~ms}$, using a neutral density filter ND3 C812579). The LED array was driven by a current of $0.3 \mathrm{~A}$; note that in the actual flight experiment, a higher current was used, which should help to reduce fluctuations and hence improve homogeneity. Measurements were taken 5 min after powering on the source to avoid startup effects.

As shown in Fig. 5, the measured light intensity across the top flat surface of the light cone is indeed homogeneous. The exemplary source used in the profiling was equipped with a hand-polished cone; the homogeneity could even further be increased by machine polishing the cone. Due to the fact that the light cone possesses a sharp edge on the boundary, some minimal fringes are seen. These are not relevant for the sample illumination since the surface of the sample cell is slightly smaller than that of the cone.

\section{B. Sample cells and imaging}

The setup uses cylindrical sample cells, with a diameter of $2 \mathrm{~mm}$ and height of $1 \mathrm{~mm}$. The sample containment consists of two glass plates, which are hydrophobized by coating with perfluorinated silanes. The sample volume is defined by a $1 \mathrm{~mm}$ thick polytetrafluoroethylene spacer with a $2 \mathrm{~mm}$ cylindrical hole. After filling, the cells are sealed by mechanical pressure. To this end, they are embedded in a housing that is thermally monitored and controlled by using a onewire thermosensor and a Peltier element. Precise thermal control is important in our samples because one needs to ensure that solvent demixing only occurs locally and due to the heating of the caps of the Janus particles. The sample housing is shown in Fig. 6. Both the temperature readings and the control of the Peltier elements to the desired accuracy of $\pm 0.5^{\circ} \mathrm{C}$ are implemented using the Raspberry Pi attached to the individual sample environment.

As described above, the sounding rocket spins during the startup phase, causing centrifugal forces on the particles that would cause them to accumulate on the side wall of the cylindrical sample cells. To counteract this effect, each sample cell is placed on a mechanical swing mechanism. During the ascent of the rocket, the bottom part of the module chamber that contains the illumination units including the light cones is lowered such that the swings can deflect freely under the action of centrifugal forces. After despinning of the rocket, a step motor controlled by the master Pi computer
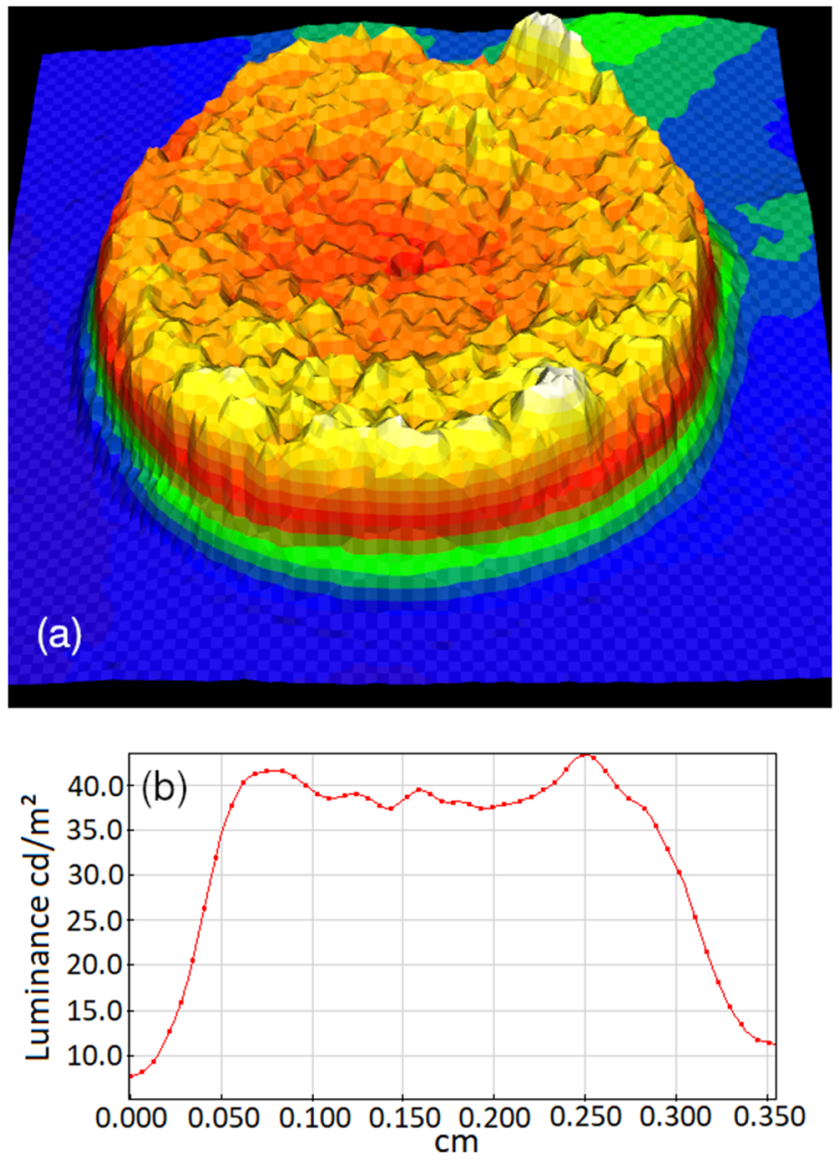

FIG. 5. Profiling of an exemplary RAMSES LED-array light source. (a) 3D plot of the relative logarithmic intensity at the upper end of the light conus. (b) Intensity trace along a radial cross section.

drives a spindle to lift the bottom part of the module chamber until the light cones come into mechanical contact with the sample cells. Through a guiding rail, it is ensured that the swings are brought back to neutral position and the sample cells are aligned with the illumination and camera axis.

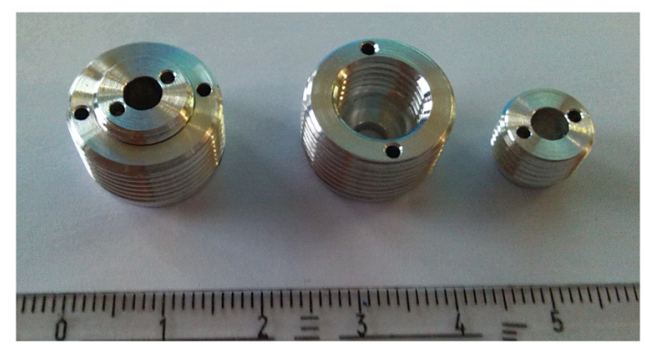

FIG. 6. Sample cells for the RAMSES setup. The left part of the image shows a closed sample holder; the middle and right parts show the two parts that are screwed together to form the sample holder and to fix the bottom glass plate, the sample with the spacer, and the cover plate in their center. 
The sample chambers are equipped with an eccentric motor that is started by the master Pi just after entering the microgravity phase and before the start of the data acquisition, to vibrate the sample cells horizontally and thus to redisperse the particles after they have sedimented due to the rocket acceleration.

The transmitted-light intensities through the sample cells are recorded by standard Raspberry Pi camera modules, modified with lenses taken from universal serial bus (USB) microscopes. The optical lengths are set up such that the camera chip (Sony IMX219 CMOS, $3280 \times 2460$ pixel $^{2}$ ) images a quarter of a sample cell, giving a resolution of $3.28 \mathrm{pixel} / \mu \mathrm{m}$ after magnification.

\section{EXPERIMENTAL TESTS}

In the following, we describe exemplary data analysis from one of the RAMSES flights. Since in three spatial dimensions tracking of individual particles through microscopy is less straight-forward than in quasi-2D, the setup was designed with the requirements of dynamical differential microscopy (DDM) in mind. $\mathrm{DDM}^{21-23}$ is a technique that allows us to use ordinary white-light microscopy for the study of the wave-vector resolved dynamics in terms of dynamical light-intensity correlation functions.

\section{A. Measurement protocol}

For particles of an average diameter of $\sigma=2.8 \mu \mathrm{m}$, free-particle motion can be characterized by a time scale $\tau_{0}=\sigma^{2} / D_{0}$, where $D_{0}=k_{B} T / 3 \pi \eta \sigma$ is the Stokes-Einstein free diffusion coefficient of a colloidal particle. We thus estimate $\tau_{0}=\left(3 \pi \eta / k_{B} T\right) \sigma^{3} \approx 46 \mathrm{~s}$. In the tests described here, images are recorded at a frame rate of about $1 \mathrm{~Hz}$, sufficient to resolve the dynamics of the particles. The images are stored on the onboard SD cards of the Raspberry Pi's and extracted postflight for data analysis.

The measurement procedure is controlled by Python scripts running on the individual Raspberry Pi computers. The scripts are automatically started after power-on, and they start a predefined measurement protocol as soon as a lift-off signal is received via the GPIO from the rocket's service module. The scripts also allow for the manual control of the experiment for use on ground or during the countdown procedure (using Ethernet connection and a virtual desktop environment running on the Linux operating system).

Temperature control is implemented on each Raspberry Pi via a simple three-step control for the Peltier elements that are connected through the GPIO pins of the Raspberry Pi. Since during extended waiting periods and during the countdown phase the dissipation of heat inside the closed module chambers needs to be minimized, the samples are kept at a lower temperature (around $23^{\circ} \mathrm{C}$ ) and heated after lift-off in order to reach the target temperature of $28.5^{\circ} \mathrm{C}$ upon entering the microgravity flight phase. Temperature readings are recorded for postflight verification.

The scripts on the master Pi additionally controls the motor and spindle system to lock the sample-cell swings and the light cones in place once the rocket has stopped spinning.

\section{B. Image analysis}

The images recorded by the Pi cameras are analyzed postflight. They are first checked manually for obvious perturbances and against the flight time line. Images not clearly associated with quiescent or microgravity conditions are discarded. In addition, a suitable image crop is performed in order to restrict the analysis to regions near the center of the sample cells. In some of the cells, part of the cell boundary is imaged and thus provides a suitable check against possible hardware misalignment during the flight. Except for the re-entry phase, the setup is stable as expected.

Typical images appear homogeneous in the cropped region, indicating homogeneous dispersion of the particles after shaking the sample with the built-in eccentric motor (see the left image in Fig. 7). To elucidate the effect of this mechanical redispersion, we also show (the right image in Fig. 7) an image taken in the microgravity phase with a sample where the eccentric motor was not run. One notes a horizontal fringe pattern that presumably comes from layering of the particles after they have been centrifuged by the spinning rocket. These patterns are stable over the entire flight time and are effectively eliminated by the shaking procedure.

The essential idea behind dynamical differential microscopy $(\mathrm{DDM})^{21}$ is to treat the camera images that capture the near-field scattered light through the sample cell as fluctuating
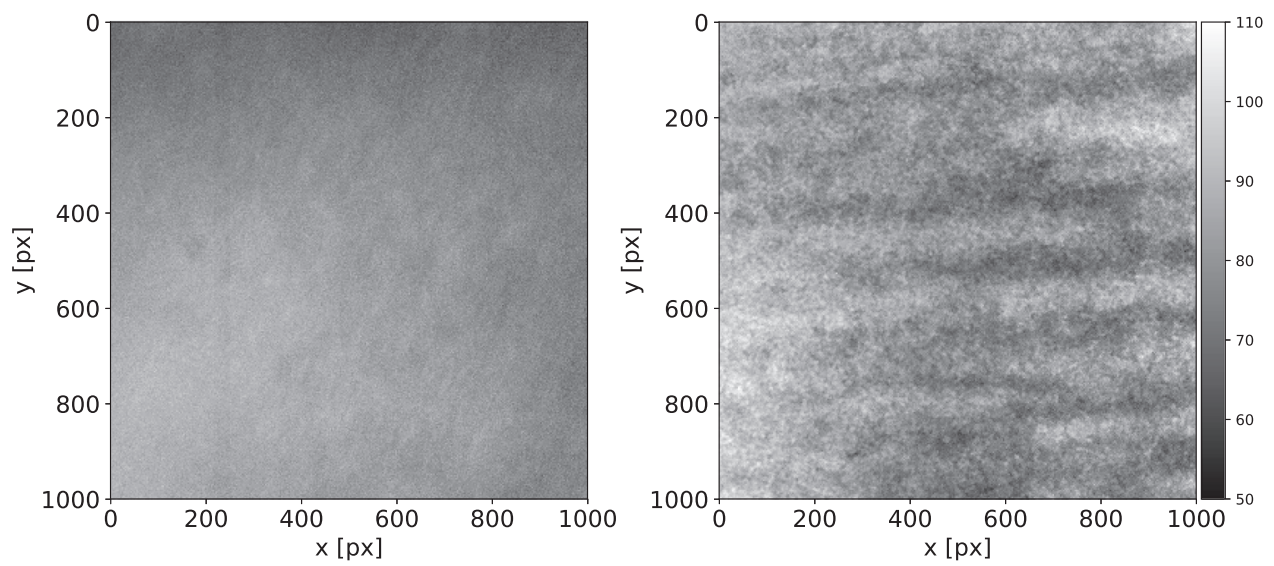

FIG. 7. Exemplary sample images recorded by the RAMSES module, cropped to the region of interest (approximately $300 \times 300 \mu \mathrm{m}^{2}$ ), for a sample consisting of $1.8 \mu \mathrm{m}$ Janus particles (nominal volume fraction $35 \%$ ). Both images are taken directly after entering the microgravity phase of the flight. Left: the sample cell after redispersion with the eccentric motor. Right: the sample cell without mechanical redispersion. Image intensity values (in the range $0-255$ ) are mapped to gray scales as indicated by the grayscale bar. 

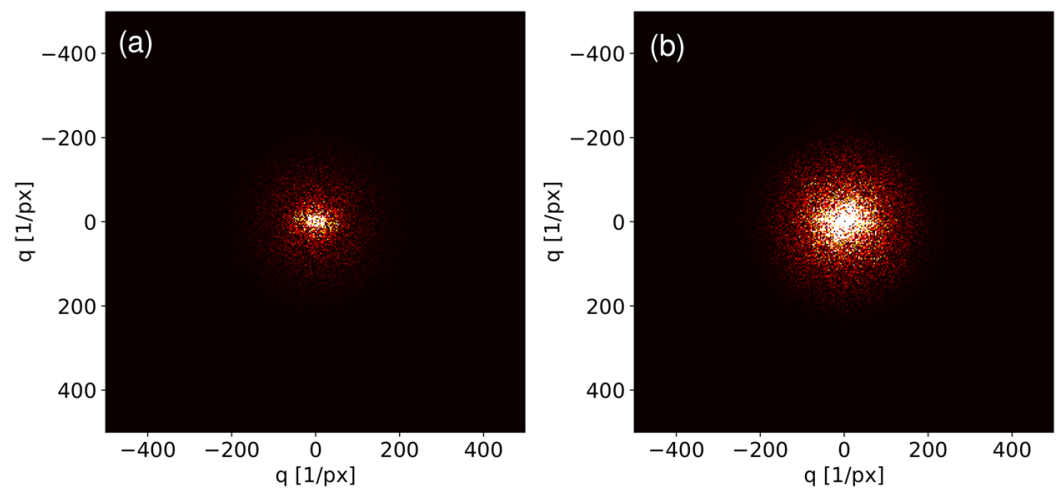

FIG. 8. Sequence of Fourier-transformed difference images from an exemplary sample taking during RAMSES' second flight: $\Delta I(\vec{r}, \Delta t, 0)$ for (a) $\Delta t=2 \mathrm{~s}$ and (b) $20 \mathrm{~s}$. speckle patterns whose Fourier transform can be related to the intensity-autocorrelation function that one would measure in a conventional dynamical-light scattering setup. A low-coherence light source minimized interference fringes in thin sample cells, and taking the Fourier transform of difference images removes static scattering coming from dust, scratches on the sample cell, or other static imperfections of the setup. The central quantity of the analysis is thus the two-dimensional intensity pattern $I(\vec{r}, t)$, from which one forms intensity differences with a time delay $\Delta t$, $\Delta I\left(\vec{r}, \Delta t, t_{0}\right)=I\left(\vec{r}, t_{0}+\Delta t\right)-I\left(\vec{r}, t_{0}\right)$, and the respective Fourier transforms, $\Delta \hat{I}\left(\vec{q}, \Delta t, t_{0}\right)=(1 / 2 \pi) \iint \Delta I\left(\vec{r}, \Delta t, t_{0}\right) \exp [-i \vec{q} \cdot \vec{r}] d^{2} r$. For a detailed discussion of the DDM analysis and a pedagogical introduction, we refer to Ref. 23, where also the code is provided that forms the basis of our analysis routines.

Typical Fourier-transformed difference images taken with the RAMSES setup are shown in Fig. 8. Here, we show a sequence of increasing time delay $\Delta t$, using the first valid frame (start of the microgravity phase) as a reference time $t_{0}=0$. We have also checked for the stationarity of the dynamics by verifying that in the cases discussed here, the image differences at fixed $\Delta t$ do not depend statistically significantly on the initial point $t_{0}$. To improve the statistics, one thus averages the resulting Fourier transforms $\Delta \hat{I}\left(\vec{q}, \Delta t, t_{0}\right)$ over a set of initial points; we typically average over $t_{0}$ distributed across the first $50 \mathrm{~s}$ of the flight, which improves the signal-to-noise ratio sufficiently while still allowing us to discuss any possible slow nonstationary aspects of the dynamics. From Fig. 8, one also checks that the dynamics is sufficiently isotropic since the Fourier transforms display the expected radial symmetry.

One thus performs radial averaging over $\vec{q}$ and obtains the DDM image correlation function $\mathcal{D}(q, \Delta t)=\left\langle\left|\Delta \hat{I}\left(\vec{q}, \Delta t, t_{0}\right)\right|^{2}\right\rangle_{q=|\vec{q}|, t_{0}}$. The principle behind DDM is that in this quantity, the static scattering contributions (in particular, also the ones from dust or imperfections in the setup) cancel out and the remaining signal is composed of the dynamical cross correlations and a static noise term,

$$
\begin{aligned}
\mathcal{D}(q, \Delta t)= & \left\langle\left|\hat{I}\left(\vec{q}, t_{0}+\Delta t\right)\right|^{2}\right\rangle_{t_{0}+\Delta t}+\left\langle\left|\hat{I}\left(\vec{q}, t_{0}\right)\right|^{2}\right\rangle_{t_{0}} \\
& -2 \operatorname{Re}\left\langle\hat{I}^{*}\left(\vec{q}, t_{0}\right) \hat{I}\left(\vec{q}, t_{0}+\Delta t\right)\right\rangle_{t_{0}}+B(q) \\
= & A(q)[1-g(q, \Delta t)]+B(q),
\end{aligned}
$$

where $g(q, \Delta t)=G(q, \Delta t) / G(q, 0)$, with $G(q, \Delta t)=\operatorname{Re}\left\langle\hat{I}^{*}\left(\vec{q}, t_{0}\right) \hat{I}\left(\vec{q}, t_{0}\right.\right.$ $+\Delta t)\rangle$ being the normalized intensity autocorrelation function that describes the colloidal dynamics of interest. $A(q)$ is a static contribution that is determined by the scattering properties of the combination of the sample, microscope optics, and illumination system. The term $B(q)$ is added $a d h o c$ as a noise term that accounts for detection noise.

The main routes of DDM data analysis ${ }^{23}$ are to extract the unknown terms $A(q)$ and $B(q)$ either from the data at sufficiently short and long time lags, as-assuming ergodic dynamics-one has $\mathcal{D}(q, 0)=B(q)$ and $\mathcal{D}(q, \infty)=A(q)+B(q)$, or from fits that usually assume a known functional form of $g(q, \Delta t)$, e.g., if the dynamics is expected to be purely diffusive, $g(q, \Delta t)=\exp \left[-q^{2} D \Delta t\right]$. We show in the following such tests of our setup.

In Fig. 9, data obtained from a 1\% volume-fraction suspension of PS particles (diameter $1 \mu \mathrm{m}$ ) are shown. The DDM data were converted to $g(q, \Delta t)$ after obtaining $A(q)$ and $B(q)$ from fits to $\mathcal{D}(q, \Delta t)$, assuming exponential relaxation. The converted data are shown as functions of diffusion time $q^{2} D_{0} \Delta t$. The data are compatible with the expected decay assuming $D_{0} \approx 0.44 \mu \mathrm{m}^{2} / \mathrm{s}$ (the expected Stokes-Einstein diffusion coefficient for $1 \mu \mathrm{m}$ sized particles).

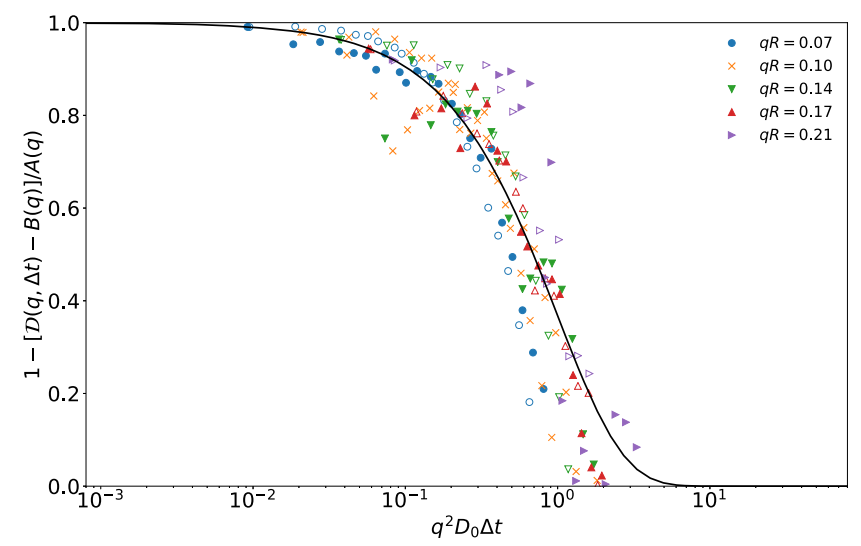

FIG. 9. Normalized intensity autocorrelation function $g(q, \Delta t)$ obtained from the DDM analysis of a sample cell using passive PS particles (average radius $R=500$ $\mathrm{nm}$, nominal volume fraction $1 \%$ ) as a function of diffusion time $q^{2} D_{0} \Delta t$, for various $q$ (different labels) Filled symbols correspond to data obtained under microgravity conditions (MAPHEUS-08 flight) and open symbols to those under $1 \mathrm{~g}$ conditions (measured during the countdown). The solid line indicates exponential decay. 


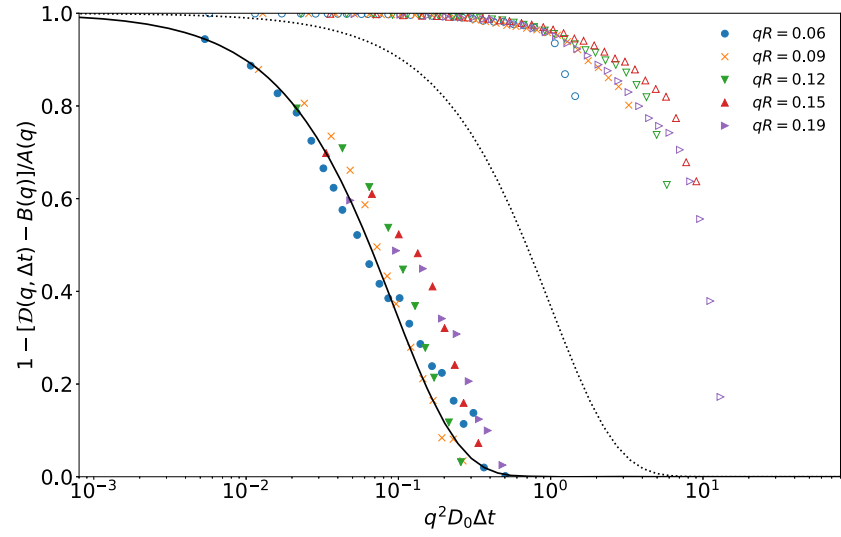

FIG. 10. Normalized intensity autocorrelation function $g(q, \Delta t)$ obtained from the DDM analysis of a sample cell using active Janus particles (average radius $R=1.4 \mu \mathrm{m}$ and nominal volume fraction $10 \%$ ), as a function of diffusion time $q^{2} D_{0} \Delta t$, for various $q$ (different labels) Filled symbols correspond to data obtained under microgravity conditions (MAPHEUS-08 flight) and open symbols to those under $1 \mathrm{~g}$ conditions (measured during the countdown). The dotted line indicates exponential decay with $D_{0}$ set by the Stokes-Einstein diffusivity for passive particles; the solid line indicates exponential decay with $D_{\text {eff }}=30 D_{0}$.

The buoyancy matching of the PS particles is sufficient such that also under $1 \mathrm{~g}$ conditions (open symbols in Fig. 9), the same diffusive dynamics is obtained as in microgravity (filled symbols). We attribute the scattering of the data around the expected exponential decay, visible in Fig. 9 for both datasets, to the fact that the optical contrast in this very dilute PS sample is rather low so that the signal-to-noise ratio obtained by the Raspberry camera module optics (which was adjusted with the optically denser Janus-particle suspensions in mind) is very low.

Figure 10 shows the corresponding $g(q, \Delta t)$ for a dilute suspension of active particles. As expected, for the low- $q$ range shown in this figure, the dynamics of the active particles is diffusive with an effective diffusion coefficient $D_{\text {eff }}=D_{0}(1+P e)$, where $P e$ is the Péclet number quantifying the activity of the particles. Our data obtained under microgravity conditions (solid symbols) are compatible with this law with $D_{\text {eff }} \approx 30 D_{0}$ (the solid line in Fig. 10). The same sample under $1 \mathrm{~g}$ conditions shows much slower dynamics (open symbols in this figure), verifying that our setup indeed captures the change in the dynamics between $1 \mathrm{~g}$ and $\mu \mathrm{g}$.

\section{DISCUSSION}

We have presented a compact setup to perform imaging experiments on three-dimensional samples of light-driven colloidal Janus particles on ground and under microgravity conditions. For the latter, the setup is designed as a highly automated and remotecontrollable module for the MAPHEUS sounding-rocket program. In order to allow systematic variation of physical model parameters, RAMSES is designed to carry ten sample cells that are all independent from each other in terms of illumination and data acquisition (to increase the fault tolerance of the whole experiment). All components, including power supply, light sources, sample cells with temperature control, optical diagnostics, and process control, are included in the confined space of a single rocket module.

Guided by the requirements of the sounding rocket flight, the RAMSES module implements a number of changes compared to the laboratory setup that can also be useful to implement there. Most notably, a new illumination system was implemented that allows us to incite self-propulsion forces through strong light fields. The illumination is based on arrays of high-power LEDs that replace conventional laser sources. They provide highly homogeneous light and allow the overall setup to be much more compact.

Second, the handling of the samples has been improved, and the combination of Teflon-coated cell walls with a purely mechanical closure (that avoids the use of glue whose components can react with lutidine as an organic solvent) has led to an improved sample stability.

Future improvements of the RAMSES setup described here include a number of technical details. The present setup was constructed to demonstrate the feasibility of microgravity experiments on a relatively short notice (less than 18 months from design to flight), using relatively cheap off-the-shelf components such as Raspberry Pi computers or USB microscopes. It provides a resolution that is sufficient to detect the active motion of the relatively large Janus particles used so far and to discern the dynamical differences in their cluster formation with and without influence of gravity. In the future, better camera systems will be used, in order to also achieve better stability of the setup to temperature fluctuations and higher frame rates to also capture the faster dynamics of potentially smaller particles and to improve the signal-to-noise ratio by acquiring more images.

As a further improvement, a more modular design of the sample cells is being developed, in order to allow a relatively easy swap between different sample environments and diagnostics. (An automated sample exchange for use on an orbital platform is also conceivable.) In combination with a sounding-rocket flight program such as MAPHEUS, which is designed to implement short turnaround times and minimizes administrative overhead, this will open the possibility of continuous access to microgravity conditions for a wide range of soft-matter experiments.

\section{ACKNOWLEDGMENTS}

The authors thank A. Meyer for his continuing support of the project and a helpful reading of the manuscript. C.B. acknowledges partial funding from the DLR e.V. Raumfahrtmanagement, section Forschung unter Weltraumbedingungen, through the Project MIKROMUG, Grant No. 50WM1950.

\section{REFERENCES}

${ }^{1}$ S. Ramaswamy, Annu. Rev. Condens. Matter Phys. 1, 323 (2010).

${ }^{2}$ J. Elgeti, R. G. Winkler, and G. Gompper, Rep. Prog. Phys. 78, 056601 (2015).

${ }^{3}$ S. Ramaswamy, J. Stat. Mech.: Theory Exp. 2017, 054002.

${ }^{4}$ J. R. Howse, R. A. L. Jones, A. J. Ryan, T. Gough, R. Vafabakhsh, and R. Golestanian, Phys. Rev. Lett. 99, 048102 (2007).

${ }^{5}$ L. Baraban, M. Tasinkevych, M. N. Popescu, S. Sanchez, S. Dietrich, and O. G. Schmidt, Soft Matter 8, 48 (2012).

${ }^{6}$ P. Romanczuk, M. Bär, W. Ebeling, B. Lindner, and L. Schimansky-Geier, Eur. Phys. J.: Spec. Top. 202, 1 (2012). 
${ }^{7}$ J. Bialké, T. Speck, and H. Löwen, Phys. Rev. Lett. 108, 168301 (2012).

${ }^{8}$ Y. Fily and M. C. Marchetti, Phys. Rev. Lett. 108, 235702 (2012).

${ }^{9}$ L. Berthier and J. Kurchan, Nat. Phys. 9, 310 (2013).

${ }^{10}$ R. Ni, M. A. C. Stuart, and M. Dijkstra, Nat. Commun. 4, 2704 (2013).

${ }^{11}$ M. E. Cates and J. Tailleur, Annu. Rev. Condens. Matter Phys. 6, 219 (2015).

${ }^{12}$ J. T. Siebert, J. Letz, T. Speck, and P. Virnau, Soft Matter 13, 1020 (2017).

${ }^{13}$ C. Bechinger, R. Di Leonardo, H. Löwen, C. Reichhardt, G. Volpe, and G. Volpe, Rev. Mod. Phys. 88, 045006 (2016).

${ }^{14}$ A. Zöttl and H. Stark, Phys. Rev. Lett. 112, 118101 (2014).

${ }^{15}$ S. Das, A. Garg, A. I. Campbell, J. Howse, A. Sen, D. Velegol, R. Golestanian, and S. J. Ebbens, Nat. Commun. 6, 8999 (2015).

${ }^{16}$ M. Enculescu and H. Stark, Phys. Rev. Lett. 107, 058301 (2011).
${ }^{17}$ G. Volpe, I. Buttinoni, D. Vogt, H.-J. Kümmerer, and C. Bechinger, Soft Matter 7, 8810 (2011).

${ }^{18}$ I. Buttinoni, G. Volpe, F. Kümmel, G. Volpe, and C. Bechinger, J. Phys.: Condens. Matter 24, 284129 (2012).

${ }^{19}$ J. R. Gomez-Solano, S. Samin, C. Lozano, P. Ruedas-Batuecas, R. van Roij, and C. Bechinger, Sci. Rep. 7, 14891 (2017).

${ }^{20}$ I. Buttinoni, J. Bialké, F. Kümmel, H. Löwen, C. Bechinger, and T. Speck, Phys. Rev. Lett. 110, 238301 (2013).

${ }^{21}$ R. Cerbino and V. Trappe, Phys. Rev. Lett. 100, 188102 (2008).

${ }^{22}$ F. Giavazzi, D. Brogioli, V. Trappe, T. Bellini, and R. Cerbino, Phys. Rev. E 80, 031403 (2009).

${ }^{23}$ D. Germain, M. Leocmach, and T. Gibaud, Am. J. Phys. 84, 202 (2016). 